\title{
Telomere Shortening in Peripheral Leukocytes Is Associated With Poor Survival in Cancer Patients Treated With Immune Checkpoint Inhibitor Therapy
}

OPEN ACCESS

Edited by:

Sebastian Kobold,

LMU Munich University Hospital,

Germany

Reviewed by:

Heinz Laubli,

University Hospital of Basel,

Switzerland

Jörg Wischhusen,

University Hospital Würzburg,

Germany

*Correspondence:

Tom Luedde

luedde@hhu.de

${ }^{t}$ These authors share first authorship

*These authors share senior authorship

Specialty section: This article was submitted to

Cancer Immunity and Immunotherapy,

a section of the journal

Frontiers in Oncology

Received: 22 June 2021 Accepted: 03 August 2021 Published: 19 August 2021

Citation:

Rolles B, Gorgulho J, Tometten M, Roderburg C, Vieri M, Abels A, Vucur M, Heymann F, Tacke $F$,

Brümmendorf TH, Luedde T, Beier F and Loosen SH (2021) Telomere Shortening in Peripheral Leukocytes is

Associated With Poor Survival in

Cancer Patients Treated With Immune

Checkpoint Inhibitor Therapy.

Front. Oncol. 11:729207. doi: 10.3389/fonc.2021.729207
Benjamin Rolles ${ }^{1,2 \dagger}$, Joao Gorgulho ${ }^{3 \dagger}$, Mareike Tometten ${ }^{1,2}$, Christoph Roderburg ${ }^{4}$, Margherita Vieri ${ }^{1,2}$, Anne Abels ${ }^{1,2}$, Mihael Vucur ${ }^{4}$, Felix Heymann ${ }^{5}$, Frank Tacke ${ }^{5}$, Tim H. Brümmendorf ${ }^{1,2}$, Tom Luedde ${ }^{4 *}$, Fabian Beier ${ }^{1,2 \ddagger}$ and Sven H. Loosen ${ }^{4 \ddagger^{\prime}}$

1 Department of Hematology, Oncology, Hemostaseology and Stem Cell Transplantation, Medical Faculty, RWTH Aachen University, Aachen, Germany, ${ }^{2}$ Center for Integrated Oncology Aachen Bonn Cologne Duesseldorf (C/O ABCD), Aachen, Germany, ${ }^{3}$ Department of Oncology, Haematology and Bone Marrow Transplantation with Section Pneumology, University Hospital Hamburg-Eppendorf, Hamburg, Germany, ${ }^{4}$ Clinic for Gastroenterology, Hepatology and Infectious Diseases, University Hospital Düsseldorf, Medical Faculty of Heinrich Heine University Düsseldorf, Düsseldorf, Germany, ${ }^{5}$ Department of Hepatology and Gastroenterology, Charité Universitätsmedizin Berlin, Berlin, Germany

Background: Immune checkpoint inhibitor (ICI) therapy represents a new standard of care for an increasing number of malignancies. Nevertheless, response rates and outcome of $\mathrm{ICl}$ treatment vary between individuals and the identification of predictive markers or hints towards immune cell exhaustion during therapy has remained a major challenge. Leukocyte telomere length is an established predictive biomarker of replicative aging and cellular proliferative potential in various hematological diseases. However, its relevance in the context of $\mathrm{ICl}$ therapy has not been investigated to date. Here, we analyze the age-adapted delta telomere length $(\Delta T L)$ of peripheral leukocytes as a potential predictive and prognostic marker in patients undergoing $\mathrm{ICl}$ therapy.

Methods: Age-adapted delta telomere length $(\Delta T L)$ of 84 patients treated with ICls for solid malignancies was measured via quantitative real-time PCR. $\Delta T L$ was correlated with outcome and clinical data.

Results: $\Delta T L$ was not significantly altered between patients with different tumor entities or tumor stages and did not predict tumor response to $\mathrm{ICl}$ therapy. However, $\Delta \mathrm{TLS}$ at initiation of treatment were a prognostic marker for overall survival (OS). When using a calculated ideal cut-off value, the median OS in patients with shorter $\Delta T L$ was 5.7 months compared to 18.0 months in patients showing longer $\Delta \mathrm{TL}$. The prognostic role of ageadapted $\Delta T L$ was further confirmed by uni- and multivariate Cox-regression analyses.

Conclusion: In the present study, we demonstrate that shorter telomere lengths in peripheral blood leukocytes are associated with a significantly impaired outcome in patients receiving $\mathrm{ICl}$ therapy across different malignancies. We explain our findings by hypothesizing an older replicative age in peripheral leukocytes of patients with an impaired 
overall survival, reflected by a premature TL shortening. Whether this association is IClspecific remains unknown. Further follow-up studies are needed to provide insights about the exact mechanism of how shortened telomeres eventually affect OS and could help guiding therapeutic decisions in future.

Keywords: PD-L1, PD-1, CTLA-4, telomere length, tumor immunity

\section{INTRODUCTION}

Immune checkpoint inhibitors (ICI) have a wide range of medical applications in various malignancies including lung cancer (1), melanoma (2), renal cell carcinoma (3) and squamous-cell carcinoma of the head and neck (4). Among a large number of antibodies, nivolumab, pembrolizumab, and ipilimumab for combination therapy, represent the most frequently used checkpoint inhibitors. Local tissue cells, activated immune cells, as well as malignant cells are able to express programmed cell death ligand 1 (PD-L1) in order to influence tumor defense (5). Nivolumab and Pembrolizumab are antibodies that inhibit the programmed cell death protein 1 (PD-1) signaling, decrease PDL1- and PD-L2-mediated immune silencing and ultimately lead to an increased anti-tumoral $\mathrm{T}$ cell activity, mainly mediated by cytotoxic T lymphocytes (6). Although ICIs have shown a high clinical efficacy in several studies, not all patients benefit from this new type of immunotherapy (7). The search for biomarkers that can help predict tolerance and response (including its long-time sustainability) to this promising type of therapy is ongoing. Previously, it has been shown that increased intratumoral expression of PD-L1 predicts a better treatment response to ICIs (8). Further studies indicate that tumors with microsatellite instability (MSI) seem to be characterized by superior response to immunotherapy using ICIs (9). Other factors such as the tumoral immune cell infiltration, tumor mutational burden, and some tumor specific mutations [e.g. BRAF-mutant non-small cell lung cancer (NSCLC) (10)], have also been associated with favorable treatment response (11). Nevertheless, predictive biomarkers of response per se as well as its long-term benefits have not yet been identified (11).

Telomeres are repetitive DNA sequences located at the ends of the chromosomes. Intact telomeres are essential for genomic integrity and the maintenance of the cellular proliferation potential (12). During life, telomeres shorten with each cell division and somatic cells become senescent or undergo apoptosis once reaching a critically short TL (13). TL represents an established biomarker for the replicative history in specific tissues, turnover of hematopoietic stem cell

Abbreviations: BMI, body mass index; CR, complete response; CTCAE, common terminology for adverse events; DC, disease control; ECOG PS, eastern cooperative oncology group performance status; GI, gastrointestinal cancers; HNC, head and neck cancers; ICI, immune checkpoint inhibitor; IRAE, immune-related adverse events; $\mathrm{MM}$, malignant melanoma; $\mathrm{nDC}$, no disease control; NSCLC, non-small cell lung cancer; OS, overall survival; PD, progressive disease; PD-1, programmed cell death protein 1; PD-L1, programmed cell death ligand 1; PR, partial response; SD, stable disease; TL, telomere length; $\Delta \mathrm{TL}$, (ageadapted) delta telomere length; UC, urothelial carcinoma; UICC, union for international cancer control. populations $(14-16)$ as well as leukocyte subsets $(14,17)$ and the biological aging process of an individual organism in general (18). For various hematological diseases (13), TL has been shown to predict treatment response, such as in chronic myeloid leukemia $(19,20)$, or has been demonstrated to correlate with the response to immunosuppressive therapy in aplastic anemia $(13,21)$. However, it currently remains unknown whether the individual TL plays a role in treatment response and outcome in patients undergoing ICI therapy. Thus, the primary aim of our study was to investigate whether the TLs of peripheral blood cells in subjects undergoing ICI therapy is a possible biomarker for therapy response and overall survival (OS).

\section{MATERIALS AND METHODS}

\section{Study Design and Patient Characteristics}

84 patients receiving treatment with ICIs for solid malignancies at the interdisciplinary cancer outpatient clinic at the University Hospital Aachen of the RWTH Aachen University were included in this study between 2018 and 2020. The patient cohort was previously described to study novel biomarkers concerning ICI therapy $(22,23)$. The study protocol was approved by the ethics committee of the University Hospital RWTH Aachen, Germany (EK 206/09) and was conducted in accordance with the ethical standards laid down in the Declaration of Helsinki. Written informed consent was obtained from all patients. Detailed patient characteristics are shown in Table 1. For all patients we documented age, body-mass-index (BMI), underlying disease, disease stage, OS, objective response rates (ORR), adverse side effects, cycles and dosage of therapy as well as standard blood counts and serum markers.

\section{Blood Sample Acquisition}

Blood samples were drawn before ICI treatment initialization or after the first administration of ICI. For the isolation of peripheral blood mononuclear cells (PBMCs), we drew blood using the BD Vacutainer ${ }^{\circledR} \mathrm{CPT}^{\mathrm{TM}}$ System (Cat No.:362782, BD Bioscience, USA), and followed manufacturer's instructions for PBMC isolation (24). PBMCs were cryopreserved with $10 \%$ DMSO at $-80^{\circ} \mathrm{C}$ until use.

\section{Assessment of Tumor Response and Overall Survival}

Tumor response was evaluated by CT or MRI scan at three, six and 12 months after treatment initialization. The different tumor responses, complete response (CR), partial response (PR), stable disease $(\mathrm{SD})$ or progressive disease $(\mathrm{PD})$ were based on the 
TABLE 1 | Patient characteristics.

\begin{tabular}{|c|c|}
\hline Parameter & Study cohort \\
\hline Cancer patients, no. & 84 \\
\hline \multicolumn{2}{|l|}{ Gender [\%]: } \\
\hline male & 66.7 \\
\hline female & 33.3 \\
\hline Age, years [median and range] & $68[34-87]$ \\
\hline $\mathrm{BMI}, \mathrm{kg} / \mathrm{m}^{2}$ [median and range] & $24.2[15.9-42.3]$ \\
\hline \multicolumn{2}{|l|}{ Tumor localization [\%]: } \\
\hline NSCLC & 40.5 \\
\hline Malignant melanoma & 11.9 \\
\hline Urothelial cancer & 11.9 \\
\hline Gl cancer & 15.5 \\
\hline Head and neck cancer & 10.7 \\
\hline Others & 9.5 \\
\hline \multicolumn{2}{|l|}{ Staging [\%]: } \\
\hline UICC III & 7.3 \\
\hline UICC IV & 92.7 \\
\hline \multicolumn{2}{|l|}{$\mathrm{ICl}$ regimen [\%]: } \\
\hline Nivolumab & 57.2 \\
\hline Pembrolizumab & 28.6 \\
\hline Nivolumab + Ipilimumab & 7.1 \\
\hline Others (e.g. Avelumab, Durvalumab) & 7.1 \\
\hline \multicolumn{2}{|l|}{ Previous systemic therapy before $\mathrm{ICl}[\%]$ : } \\
\hline Yes & 70.2 \\
\hline No & 29.8 \\
\hline \multicolumn{2}{|l|}{ ECOG PS [\%]: } \\
\hline ECOG 0 & 4.8 \\
\hline ECOG 1 & 54.8 \\
\hline ECOG 2 & 36.9 \\
\hline ECOG 3 & 3.6 \\
\hline \multicolumn{2}{|l|}{ Smoking status [\%]: } \\
\hline Never & 8.3 \\
\hline Previous & 40.5 \\
\hline Present & 20.2 \\
\hline Unknown & 31.0 \\
\hline \multicolumn{2}{|l|}{ Disease control at 3 months [\%]: } \\
\hline Yes & 42.9 \\
\hline No & 57.1 \\
\hline \multicolumn{2}{|l|}{ Disease control at 6 months [\%]: } \\
\hline Yes & 33.3 \\
\hline No & 66.7 \\
\hline \multicolumn{2}{|l|}{ Disease control at 12 months [\%]: } \\
\hline Yes & 26.5 \\
\hline No & 73.5 \\
\hline \multicolumn{2}{|l|}{ Deceased during follow-up [\%]: } \\
\hline Yes & 71.4 \\
\hline No & 28.6 \\
\hline \multicolumn{2}{|l|}{ Side effects to ICI? [\%]: } \\
\hline Yes & 39.3 \\
\hline No & 60.7 \\
\hline
\end{tabular}

No, number; BMI, body mass index; NSCLC, non-small cell lung cancer; Gl, gastrointestinal; UICC, Union for International Cancer Control; ICl, immune checkpoint inhibitor; ECOG PS, Eastern Cooperative Oncology Group performance status.

evaluation of an experienced radiologist. OS was calculated from the first day of immunotherapy to death. CR, PR, and SD were subsumed as "disease control" (DC) whereas PD was classified as "no disease control" (nDC).

\section{Assessment of Immune-Related Adverse Events}

Therapy tolerance of all patients was reviewed at regular intervals by an experienced oncologist. Immune-related adverse events
(IRAEs) were separately documented and treated based on the recommendations of the American Society of Clinical Oncology (ASCO) (25). The severity was evaluated using the Common Terminology for Adverse Events (CTCAE) version 5.0.

\section{Telomere Length Measurement}

DNA from peripheral blood cells was extracted and 1.4ng of genomic DNA was used for TL analysis. Measurement of TL was performed using the Absolute Human Telomere Length Quantification qPCR Assay Kit (ScienCell, Carlsbad, CA, USA) and FastStart Essential DNA Green Master (Roche, Basel, Switzerland). Quantitative real-time PCR (qPCR) was done with an ABI7500fast real-time PCR system (Applied Biosystems, Foster city, CA, USA) according to the Assay Kit protocol and described previously (26). TL was measured as T/S ratio. Peripheral blood samples of 104 healthy blood donors of various ages were used as control group. The healthy comparison population had a mean age of 43 years. The age range of subjects was from 18 years to 83 years. TL analysis was carried out singleblinded. Linear regression analysis was used to calculate the physiological telomere shortening during aging. For ageadaptation, patients' measured TL was related to the respective calculated TL of the control population at the same age as described previously $(20,27)$. The calculated relative TL differences result in the "age-adapted delta telomere length" $(\Delta \mathrm{TL})$. Negative values correspond to a shortening of telomeres compared to the healthy reference population.

\section{Statistical Analysis}

Shapiro-Wilk-Test was used to test for normal distribution. Non-parametric data were compared by Mann-Whitney-UTest and the Kruskal-Wallis-Test. Box plot graphics display the median, quartiles and ranges. Kaplan-Meier curves show the impact of different $\Delta$ TL cut-off values on OS. The Log-rank test was used to test for statistical differences between subgroups in Kaplan-Meier curve analyses. To identify the optimal $\Delta \mathrm{TL}$ cutoff for OS, we used Cox proportional hazard models to the dichotomized survival status as well as the survival and defined the optimal cut-off as the point with the most significant split in log-rank test. The prognostic value of variables was further tested by uni- and multivariate Cox regression analyses. Parameters with a p-value of $<0.250$ in univariate testing were included into multivariate testing. The hazard ratio (HR) and the $95 \%$ confidence interval are displayed. All statistical analyses were performed with SPSS 23 (SPSS, Chicago, IL, USA) and RStudio 1.2.5033 (RStudio Inc., Boston, MA, USA) (28). P-value of $<0.05$ was considered as statistically significant $\left({ }^{*} \mathrm{p}<0.05 ;{ }^{* *} \mathrm{p}<0.01\right.$; $\left.{ }^{* * *} \mathrm{p}<0.001\right)$.

\section{RESULTS}

\section{Evaluation of TL in Relation to Patient Characteristics}

Our cohort of 84 patients was analyzed for age-adapted $\Delta \mathrm{TL}$. Clinical characteristics of the study cohort are summarized in 
Table 1. We found no differences in $\Delta \mathrm{TL}$ between patients with NSCLC, malignant melanoma (MM), urothelial carcinoma (UC), gastrointestinal cancers (GI), head and neck cancers (HNC) or other entities. There was a (non-significant) trend that $\Delta \mathrm{TL}$ tended to be shorter in patients with HNC than in patients with MM (Figure 1A). Although overall median $\Delta \mathrm{TL}$ was shortest in patients with ECOG 3 performance status, there was no significant difference between the different performance status (Figure 1B). In our cohort, smoking status had no impact on $\Delta \mathrm{TL}$, not even in actively smoking patients (Figure 1C). Furthermore, gender also had no impact on $\Delta \mathrm{TL}$ (Figure 1D). All patients had an advanced tumor stage, with 6 patients in UICC stage III and 76 patients in UICC stage IV. Within that range, the UICC stage had no impact on $\Delta \mathrm{TL}$ (Figure 1E). We also did not observe a significant difference in $\Delta \mathrm{TL}$ in patients treated with nivolumab, pembrolizumab, the combination of nivolumab and ipilimumab or other ICIs (Figure 1F). Interestingly, whether patients had received other systemic therapies before ICI initialization had also no effect on patients' $\Delta \mathrm{TL}$ (Figure 1G).

\section{Evaluation of Treatment Response to ICI Therapy}

We next analyzed whether the patients' $\Delta \mathrm{TL}$ at baseline (ICI initiation) had an impact on treatment response to ICI therapy after three, six or 12 months. When looking at treatment response at three months, we observed comparable $\Delta \mathrm{TL}$ values between patients who showed a controlled disease (DC, including patients with $\mathrm{CR}, \mathrm{PR}$, and $\mathrm{SD}$ ) and patients with progressive disease (non-DC, Figure 2A). In line, the $\Delta \mathrm{TL}$ value was unaltered between patients who did or did not show DC at six and 12 months, respectively (Figures $\mathbf{2 B}, \mathbf{C}$ ). Similar results were obtained, when applying a different categorization of tumor responders (responders: CR and PR vs. non-responders: $\mathrm{SD}$ and $\mathrm{PD})$.

\section{A Shortened $\Delta T L$ Is Associated With an Impaired Overall Survival}

Based on previous findings of TL as a biomarker for treatment response $(19,20,29)$, we next analyzed whether the individual

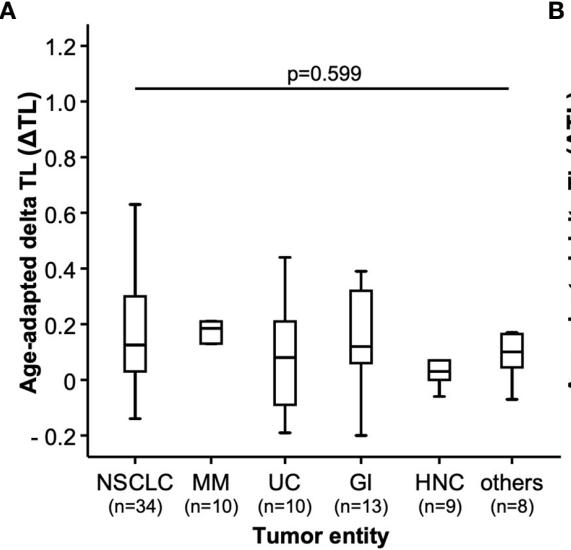

D

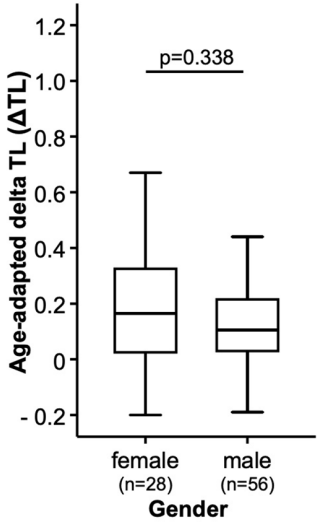

E

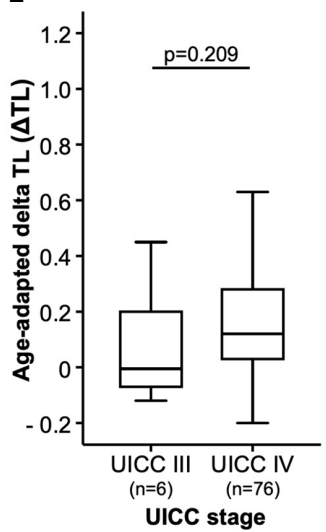

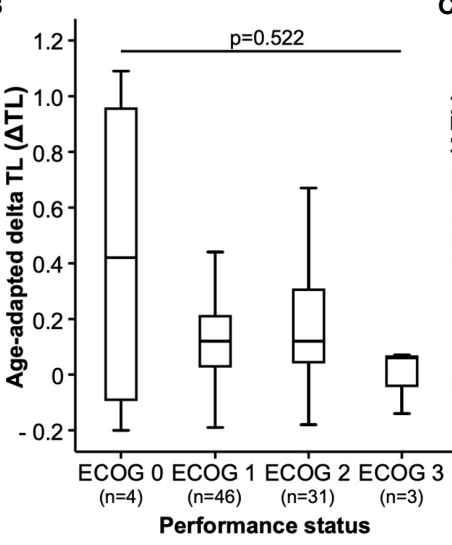

F

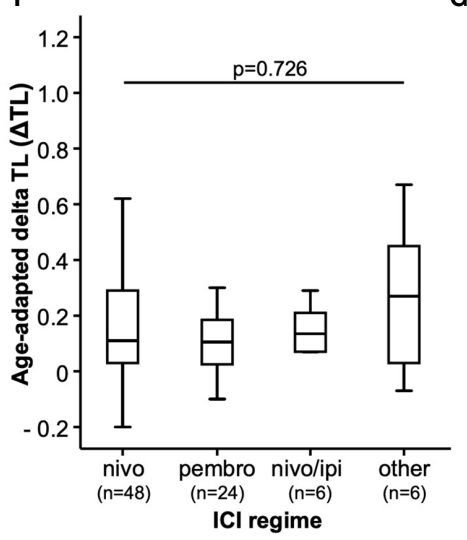

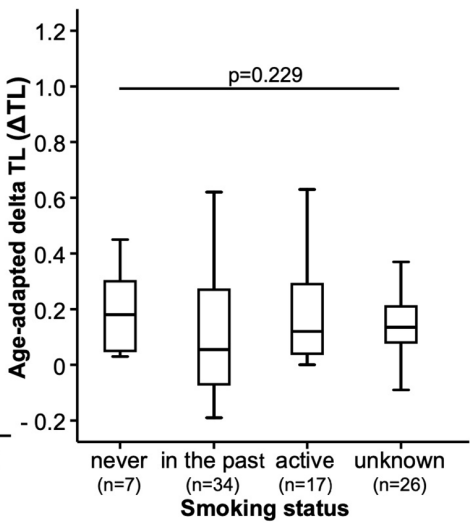

G

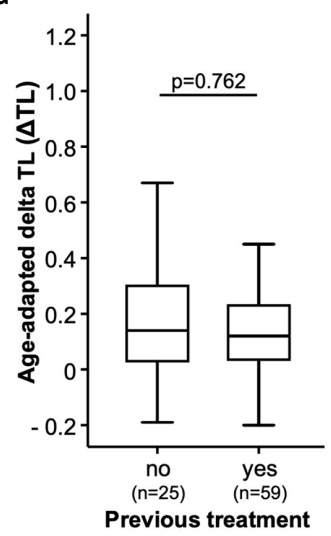

FIGURE 1 | Study population. The age-adapted delta telomere length $(\Delta T L)$ is shown in patients with different (A) tumor entities [non-small cell lung cancer (NSCLC), malignant melanoma (MM), urothelial cancer (UC), gastrointestinal cancer (GI) head and neck cancer (HNC) and other tumor types (other)], (B) ECOG performance status, (C) smoking status, (D) gender of the patient, (E) advanced tumor stages classified by the UICC, (F) type of immunotherapeutics (nivolumab (nivo), pembrolizumab (pembro), a combination of nivolumab and ipilimumab (nivo/ipi) or other immunotherapeutics (other) and (G) if patients received pre-treatments (yes) or not (no). 

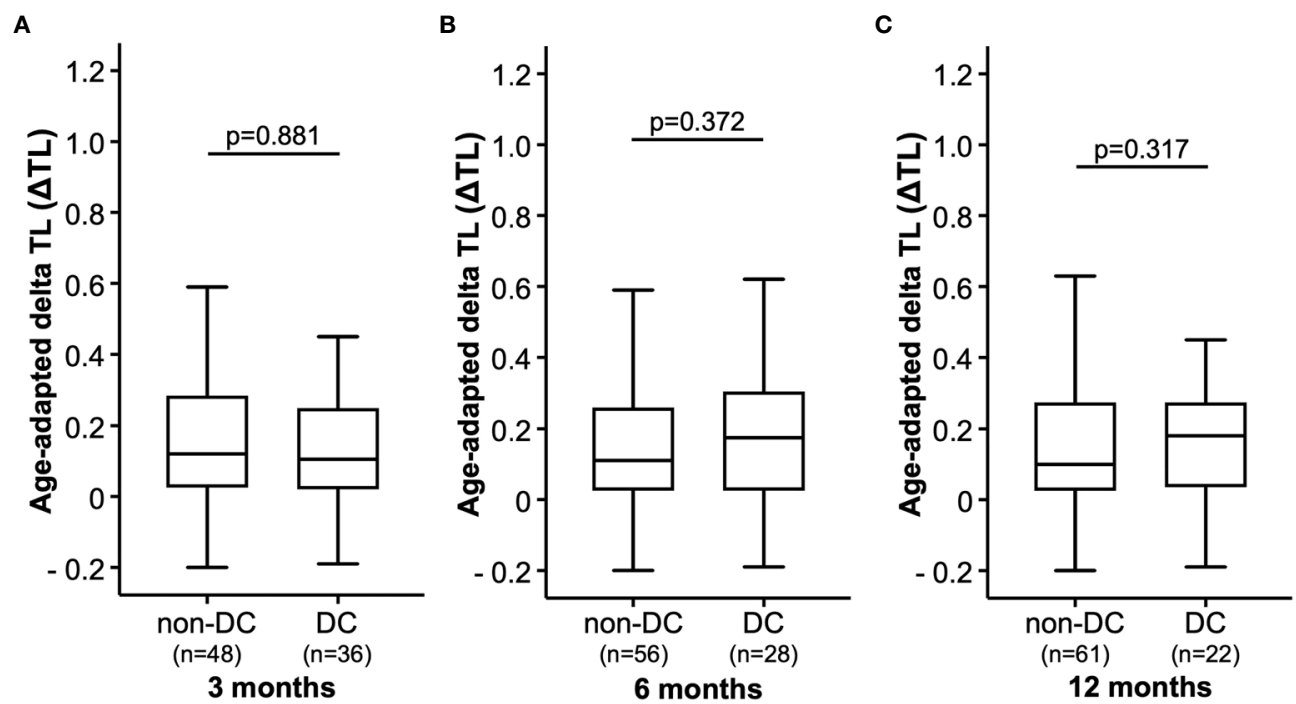

FIGURE 2 | Therapy response. The age-adapted delta telomere length ( $\Delta \mathrm{TL})$ is shown in patients with disease control (DC) or without disease control (non-DC) after (A) 3 months, (B) 6 months and (C) 9 months.

$\Delta \mathrm{TL}$ had an impact on the patients' OS. Using the median $\Delta \mathrm{TL}$ of all patients as a cut-off value (0.12), we observed a significantly impaired OS in patients with a $\Delta \mathrm{TL}$ shorter than the cut-off value (Figure 3A). Subsequently, we established an ideal prognostic cut-off value of 0.17 via determination of the most significant split in the log rank test between patients with a good or poor outcome. Using this cut-off value, Kaplan-Meier curve analysis revealed a highly significantly impaired OS in patients with a $\Delta \mathrm{TL}$ value below the ideal cut-off value (Figure 3B). The median OS in these patients was only 5.7 months compared to 18.0 months in patients with a median $\Delta \mathrm{TL}$ above the ideal cut-off value. In a next step, we performed uni- and multivariate Cox-regression analyses to further substantiate the prognostic relevance of $\Delta \mathrm{TL}$ in terms of OS. In univariate analysis, $\Delta \mathrm{TL}$ turned out as a significant prognostic marker for OS (HR: 0.295, 95\%CI: $0.092-0.950, p=0.041)$. When including other parameters of prognostic relevance in univariate analysis into multivariate Cox-regression analysis (BMI, ECOG PS, bilirubin and creatinine), the prognostic role of $\Delta \mathrm{TL}$ was found to be independent of these confounders (HR: 0.280, 95\%CI: 0.081$0.968, \mathrm{p}=0.044$, Table 2). While adaptation of TLs for age is a common practice, differences in OS remained significant even when based on non-age-adapted TLs (HR: 0.308; 95\%CI: 0.099-0.958; $\mathrm{p}=0.042$ ).

\section{IRAEs During ICI Therapy}

We finally evaluated whether the individual $\Delta \mathrm{TL}$ was associated with the occurrence of IRAEs during ICI therapy. IRAEs were observed in 33 patients (39.3\%). Of these, 9 IRAEs were classified as a severe side effect grade $\geq 3$ CTCAE. However, when we compared the initial $\Delta \mathrm{TL}$ between patients with or without
IRAE/severe IRAE, we did not observe any significant differences (Figures 4A, B).

\section{DISCUSSION}

Despite of the broad application of ICIs in various tumor entities, few factors have been established predicting treatment response and OS to ICI treatment (30). TL represents an established biomarker in various hematological diseases $(13,19-21)$. The impact of TL on the survival of patients under ICI therapy has not been investigated before. In our study, we were able to demonstrate that patients with longer age-adapted $\Delta \mathrm{TLs}$ had an improved OS compared to patients with shorter $\Delta \mathrm{TL}$, independent of the underlying malignant disease. Of note, we observed no differences in treatment response to ICI therapy with respect to patients' $\Delta$ TL. Furthermore, we were not able to demonstrate an association between $\Delta \mathrm{TL}$ and tumor type, stage of disease, age, gender, previous treatments of patients or IRAEs. We could not observe the previously described shorter $\Delta$ TLs of smokers compared to non-smokers possibly due to the small sample size (31). However, we were able to confirm previous data showing that an increased body-mass-index (BMI) had a positive impact on OS in patients receiving ICIs (32).

In the present study using univariate and multivariate analyses, we show that the individual $\Delta \mathrm{TL}$ in our cohort with heterogeneous solid tumors had a significant impact on the patients' OS. This effect was most significant when using an ideal $\Delta \mathrm{TL}$ cut-off value of 0.17 . At this cut-off value, the median OS in patients with low $\Delta \mathrm{TL}$ was only 5.7 months compared to 18.0 months in patients showing a $\Delta \mathrm{TL}$ above this cut-off value. Since $\Delta \mathrm{TL}$ is not specifically correlating with known factors for 


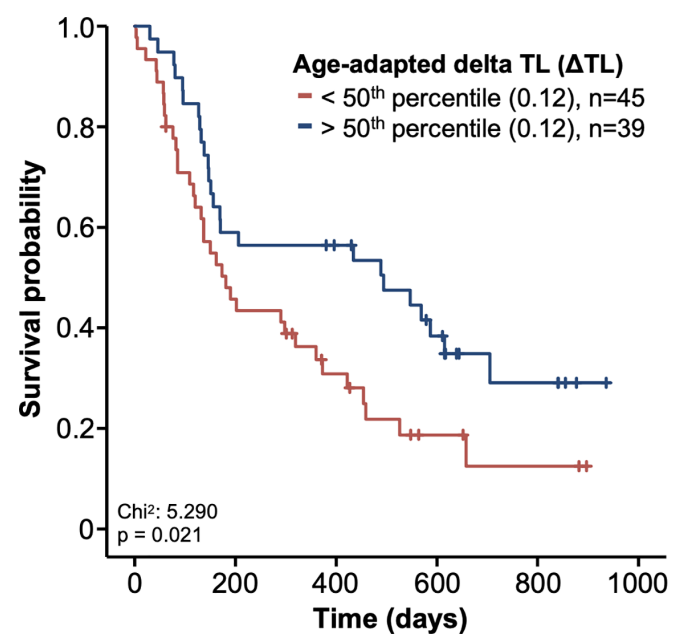

B

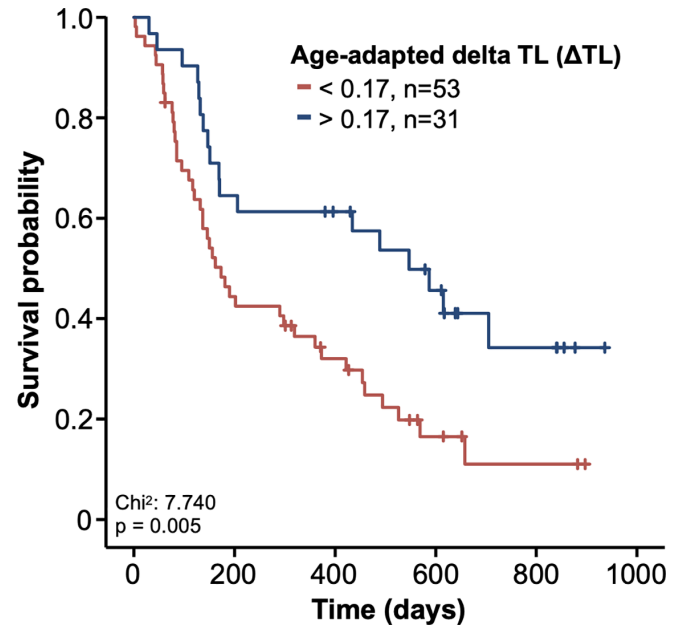

FIGURE 3 | Kaplan-Meier curves of survival. The survival probability is shown in relation to the course of time. Patients were divided into two groups based on the $\Delta T L$. (A) In the first graphic, the group was split based on the $50^{\text {th }}$ percentile. (B) In the second graphic, a relative telomere length of 0.17 was used as a cut-off. Patients with longer telomeres are shown in blue and patients with shorter telomeres are shown in red.

TABLE 2 | Uni- and multivariate Cox-regression analysis for the prediction of overall survival.

\begin{tabular}{|c|c|c|c|c|}
\hline \multirow[t]{2}{*}{ Parameter } & \multicolumn{2}{|c|}{ Univariate Cox-regression } & \multicolumn{2}{|c|}{ Multivariate Cox-regression } \\
\hline & p-value & Hazard-Ratio (95\% Cl) & p-value & Hazard-Ratio (95\% Cl) \\
\hline$\Delta \mathrm{TL}$ & 0.041 & 0.295 (0.092-0.950) & 0.044 & $0.280(0.081-0.968)$ \\
\hline Age & 0.806 & 0.997 (0.971-1.023) & & \\
\hline Sex & 0.588 & $0.863(0.506-1.470)$ & & \\
\hline BMI & 0.007 & $0.928(0.879-0.980)$ & 0.022 & $0.936(0.885-0.990)$ \\
\hline UICC tumor stage & 0.407 & $1.639(0.510-5.267)$ & & \\
\hline ECOG PS & 0.182 & $1.330(0.875-2.020)$ & 0.091 & 1.488 (0.939-2.359) \\
\hline Leukocyte count & 0.354 & 1.027 (0.970-1.088) & & \\
\hline Bilirubin & 0.127 & 1.519 (0.888-2.599) & 0.041 & $1.711(1.023-2.862)$ \\
\hline Creatinine & 0.242 & $0.755(0.472-1.08)$ & 0.691 & $0.909(0.567-1.457)$ \\
\hline
\end{tabular}

BMI, Body-Mass-Index; UICC, Union for International Cancer Control; ECOG PS, Eastern Cooperative Oncology Group performance status.

survival as e.g., tumor stage, tumor entity, performance score or response to treatment, we explain the differences in the survival curves with the fact that TLs might represent a possible marker for the assessment of biological age. Due to the relatively small subgroup sizes, no tumor entity-specific analyses were performed. Furthermore, we cannot establish a clear association with ICI therapy because we lack a comparison group receiving any other type of treatment, such as chemotherapy.

Different studies have shown in the past that telomeres shorten as part of the physiological aging process and TL of peripheral blood leukocytes represent an established global marker for the aging process of the entire organism $(12,18)$. Supporting our hypothesis of shorter TL as marker for the older biological age, previous studies demonstrated that within the general population, shortened TLs are associated with increased all-cause mortality (33). In oncological patients, a comprehensive study including over 47,000 cancer patients revealed that TL correlates with the outcome of cancer patients (34). In line with this observation, performance status scales like "ECOG" or the
"Karnofsky-Index" as an indirect marker for biological fitness were shown to predict survival in oncological patients (35). Furthermore, it was previously shown that also immune cell ageing based on different markers was associated with increased mortality (36). We therefore speculate that shorter TLs will also be associated with an increased non-tumor related mortality in our collective as a possible cause for observed difference in OS. This effect could be amplified by pre-existing diseases and tumor-related complications. However, we have insufficient information regarding the specific cause of death of our patients or detailed information about patient's pre-existing diseases to definitively prove our hypothesis.

An open question in the field is whether additional factors can specifically cause TL shortening in peripheral blood leukocytes. A variety of environmental factors or chronic diseases can in addition accelerate the physiological telomere related aging process in leukocytes $(37,38)$. In addition, tumor proinflammatory milieu and its microenvironment may also contribute to TL shortening. Data suggest that tumor-associated 


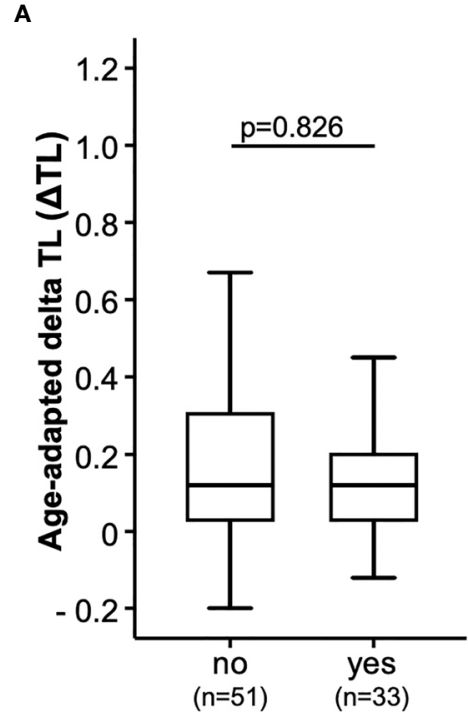

IRAE

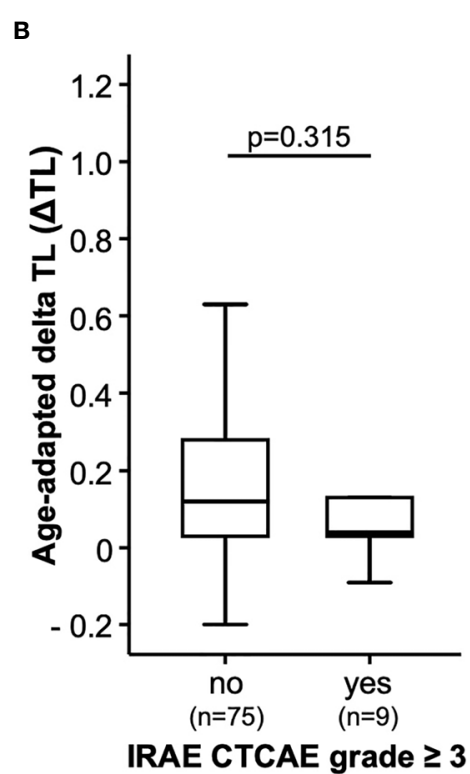

FIGURE 4 | Immune-related adverse events during immune checkpoint inhibitor therapy. The age-adapted delta telomere length ( $\Delta$ TL) is shown (A) in a group of patients without (no) or with (yes) immune-related adverse events (IRAEs). (B) In addition, $\Delta T L$ is shown in patients with severe IRAEs > grade III (yes) and in those patients who do not (no).

inflammation can lead to age-independent immune senescence with consecutive shortening of the TL $(39,40)$. Due to the old age of our cohort, congenital defects in telomerase-associated proteins leading to impaired telomere maintenance seem to be unlikely as major factor.

Lymphocytes are crucial cells for tumor defense and are substantially involved in maintaining an anti-tumoral immune response, mainly within the context of ICI therapy (1). It is tempting to speculate that premature aging in the $\mathrm{T}$-cell compartment results in immune cell exhaustion explaining the impaired OS in patients with shorter TL (41). This issue becomes of particular interest since preliminary work has shown that telomere shortening can predominantly occur in specific immune cell subpopulations, e.g., in memory cytotoxic $\mathrm{T}$ cells (42). Due to methodological constraints, we only measured $\Delta \mathrm{TL}$ of all leukocytes, and it is quite possible that important differences in $\Delta \mathrm{TL}$ that may predict treatment efficacy are only evident in subpopulations, such as lymphocytes. In the case of immune cell exhaustion, we would first expect a shortening of the $\Delta \mathrm{TL}$ in the lymphocyte compartment. However, it is therefore all the more surprising that we can already detect differences in survival in a small cohort and with a global screening of $\Delta T L$. Although our study did not demonstrate an inferior response to ICI in patients with shorter TL, the significantly shortened survival time indicates that we may not have captured (small) differences in treatment response or IRAEs due the small patient cohort as well as the selection bias of our patient population. It is possible that subtle differences may be hidden by our basked study design.
Our study has some limitations as the analyzed patient cohort is heterogenous including patients with different tumor entities who receive different ICIs. We also did not include patients who were treated with different therapeutic approaches such as chemotherapy, radiotherapy, or surgery. Moreover, validation of our exploratory findings in larger patient cohorts, that we hope to have stimulated with our study, is essential before a potential clinical implementation can be considered.

\section{CONCLUSION}

Together, in a collective of 84 patients who underwent therapy with ICIs, we observed that $\triangle \mathrm{TL}$ at treatment initialization was an independent predictor of OS. We explain our findings with a hypothetical older biological age represented by a premature TL shortening. The pathophysiological background of our observation appears to be unclear. An impact of TL on therapy response or on therapy-associated side effects could not be proven. Additional studies are needed to analyze if further mechanisms that lead to TL shortening in patients with solid tumors exist and to find out if long TLs, especially in lymphocytes, support immunosurveillance competence under ICI therapy during long-term observations.

\section{DATA AVAILABILITY STATEMENT}

The raw data supporting the conclusions of this article will be made available by the authors, without undue reservation. 


\section{ETHICS STATEMENT}

The studies involving human participants were reviewed and approved by Ethics committee of the University Hospital RWTH Aachen, Germany (approval EK 206/09). The patients/participants provided their written informed consent to participate in this study.

\section{AUTHOR CONTRIBUTIONS}

SL, TL, BR, and FB designed the study. JG and SL recruited patients. BR and AA performed experiments. SL, JG, FB, and BR performed statistical analysis and generated Figures and Tables. $\mathrm{MT}, \mathrm{CR}, \mathrm{MV}, \mathrm{FT}, \mathrm{FH}, \mathrm{TB}$, and TL provided intellectual input. $\mathrm{BR}, \mathrm{SL}, \mathrm{JG}, \mathrm{FB}$, and TL drafted the manuscript. SL, BR, FB, and JG revised the manuscript. All authors contributed to the article and approved the submitted version.

\section{REFERENCES}

1. Waldman AD, Fritz JM, Lenardo MJ. A Guide to Cancer Immunotherapy: From T Cell Basic Science to Clinical Practice. Nat Rev Immunol (2020) 20:651-68. doi: 10.1038/s41577-020-0306-5

2. Larkin J, Chiarion-Sileni V, Gonzalez R, Grob J-J, Rutkowski P, Lao CD, et al. Five-Year Survival With Combined Nivolumab and Ipilimumab in Advanced Melanoma. N Engl J Med (2019) 381:1535-46. doi: 10.1056/NEJMoa1910836

3. Motzer RJ, Rini BI, McDermott DF, Arén Frontera O, Hammers HJ, Carducci MA, et al. Nivolumab Plus Ipilimumab Versus Sunitinib in First-Line Treatment for Advanced Renal Cell Carcinoma: Extended Follow-Up of Efficacy and Safety Results From a Randomised, Controlled, Phase 3 Trial. Lancet Oncol (2019) 20:1370-85. doi: 10.1016/S1470-2045(19)30413-9

4. Ferris RL, Blumenschein G, Fayette J, Guigay J, Colevas AD, Licitra L, et al. Nivolumab for Recurrent Squamous-Cell Carcinoma of the Head and Neck. N Engl J Med (2016) 375:1856-67. doi: 10.1056/NEJMoa1602252

5. Sharpe AH, Pauken KE. The Diverse Functions of the PD1 Inhibitory Pathway. Nat Rev Immunol (2018) 18:153-67. doi: 10.1038/nri.2017.108

6. Alsaab HO, Sau S, Alzhrani R, Tatiparti K, Bhise K, Kashaw SK, et al. PD-1 and PD-L1 Checkpoint Signaling Inhibition for Cancer Immunotherapy: Mechanism, Combinations, and Clinical Outcome. Front Pharmacol (2017) 8:561. doi: 10.3389/fphar.2017.00561

7. Jenkins RW, Barbie DA, Flaherty KT. Mechanisms of Resistance to Immune Checkpoint Inhibitors. Br J Cancer (2018) 118:9-16. doi: 10.1038/ bjc. 2017.434

8. Sun C, Mezzadra R, Schumacher TN. Regulation and Function of the PD-L1 Checkpoint. Immunity (2018) 48:434-52. doi: 10.1016/j.immuni.2018.03.014

9. Ding L, Chen F. Predicting Tumor Response to PD-1 Blockade. N Engl J Med (2019) 381:477-9. doi: 10.1056/NEJMcibr1906340

10. Dudnik E, Peled N, Nechushtan H, Wollner M, Onn A, Agbarya A, et al. BRAF Mutant Lung Cancer: Programmed Death Ligand 1 Expression, Tumor Mutational Burden, Microsatellite Instability Status, and Response to Immune Check-Point Inhibitors. J Thorac Oncol (2018) 13:1128-37. doi: 10.1016/ j.jtho.2018.04.024

11. Havel JJ, Chowell D, Chan TA. The Evolving Landscape of Biomarkers for Checkpoint Inhibitor Immunotherapy. Nat Rev Cancer (2019) 19:133-50. doi: 10.1038/s41568-019-0116-x

12. Blasco MA. Telomere Length, Stem Cells and Aging. Nat Chem Biol (2007) 3:640-9. doi: 10.1038/nchembio.2007.38

13. Brümmendorf $\mathrm{TH}$, Balabanov S. Telomere Length Dynamics in Normal Hematopoiesis and in Disease States Characterized by Increased Stem Cell Turnover. Leukemia (2006) 20:1706-16. doi: 10.1038/sj.leu.2404339

14. Rufer N, Brümmendorf TH, Kolvraa S, Bischoff C, Christensen K, Wadsworth $\mathrm{L}$, et al. Telomere Fluorescence Measurements in Granulocytes and $\mathrm{T}$ Lymphocyte Subsets Point to a High Turnover of Hematopoietic Stem Cells

\section{FUNDING}

This publication is part of a project that has received funding from the European Research Council (ERC) under the European Union's Horizon 2020 research and innovation program (Grant agreement No. 771083). The lab of TL was further supported by the German Cancer Aid (Deutsche Krebshilfe 110043 and a Mildred-Scheel-Professorship), the German-ResearchFoundation (SFB-TRR57/P06, LU 1360/3-1, CRC1380/A01, and CA 830/3-1), the Ernst-Jung-Foundation Hamburg, the IZKF (interdisciplinary centre of clinical research) Aachen and a grant from the medical faculty of the RWTH Aachen. Margherita Vieri receives funding from the "Aachener Krebsund Leukämiehilfe". FB was funded by a START Grant (No. 691743, RWTH Aachen University) and part of the work was supported by a grant of the "Württembergischer Krebspreis 2019 " to FB.

and Memory T Cells in Early Childhood. J Exp Med (1999) 190:157-67. doi: 10.1084/jem.190.2.157

15. Brümmendorf TH, Mak J, Sabo KM, Baerlocher GM, Dietz K, Abkowitz JL, et al. Longitudinal Studies of Telomere Length in Feline Blood Cells. Exp Hematol (2002) 30:1147-52. doi: 10.1016/S0301-472X(02)00888-3

16. Werner B, Beier F, Hummel S, Balabanov S, Lassay L, Orlikowsky T, et al. Reconstructing the In Vivo Dynamics of Hematopoietic Stem Cells From Telomere Length Distributions. Elife (2015) 4:e08687. doi: 10.7554/eLife.08687

17. Baerlocher GM, Lansdorp PM. Telomere Length Measurements in Leukocyte Subsets by Automated Multicolor Flow-FISH. Cytomet A (2003) 55:1-6. doi: 10.1002/cyto.a.10064

18. López-Otín C, Blasco MA, Partridge L, Serrano M, Kroemer G. The Hallmarks of Aging. Cell (2013) 153:1194-217. doi: 10.1016/j.cell.2013.05.039

19. Bouillon A-S, Ventura Ferreira MS, Awad SA, Richter J, Hochhaus A, Kunzmann V, et al. Telomere Shortening Correlates With Leukemic Stem Cell Burden at Diagnosis of Chronic Myeloid Leukemia. Blood Adv (2018) 2:1572-9. doi: 10.1182/bloodadvances.2018017772

20. Wenn K, Tomala L, Wilop S, Vankann L, Hasenbank C, Frank O, et al. Telomere Length at Diagnosis of Chronic Phase Chronic Myeloid Leukemia (CML-CP) Identifies a Subgroup With Favourable Prognostic Parameters and Molecular Response According to the ELN Criteria After 12 Months of Treatment With Nilotinib. Leukemia (2015) 29:2402-4. doi: 10.1038/ leu.2015.245

21. Brümmendorf TH, Maciejewski JP, Mak J, Young NS, Lansdorp PM. Telomere Length in Leukocyte Subpopulations of Patients With Aplastic Anemia. Blood (2001) 97:895-900. doi: 10.1182/blood.v97.4.895

22. Loosen SH, Gorgulho J, Jördens MS, Schulze-Hagen M, Beier F, Vucur M, et al. Serum Levels of Soluble Urokinase Plasminogen Activator Receptor Predict Tumor Response and Outcome to Immune Checkpoint Inhibitor Therapy. Front Oncol (2021) 11:646884. doi: 10.3389/fonc.2021.646883

23. Loosen SH, van den Bosch V, Gorgulho J, Schulze-Hagen M, Kandler J, Jördens MS, et al. Progressive Sarcopenia Correlates With Poor Response and Outcome to Immune Checkpoint Inhibitor Therapy. J Clin Med (2021) 10:1361. doi: $10.3390 / \mathrm{jcm} 10071361$

24. Ruitenberg JJ, Mulder CB, Maino VC, Landay AL, Ghanekar SA. VACUTAINER CPT and Ficoll Density Gradient Separation Perform Equivalently in Maintaining the Quality and Function of PBMC From HIV Seropositive Blood Samples. BMC Immunol (2006) 7:11. doi: 10.1186/1471-2172-7-11

25. Brahmer JR, Lacchetti C, Schneider BJ, Atkins MB, Brassil KJ, Caterino JM, et al. Management of Immune-Related Adverse Events in Patients Treated With Immune Checkpoint Inhibitor Therapy: American Society of Clinical Oncology Clinical Practice Guideline. J Clin (2018) 36:1714-68. doi: 10.1200/ JCO.2017.77.6385

26. Vieri M, Kirschner M, Tometten M, Abels A, Rolles B, Isfort S, et al. Comparable Effects of the Androgen Derivatives Danazol, Oxymetholone 
and Nandrolone on Telomerase Activity in Human Primary Hematopoietic Cells From Patients With Dyskeratosis Congenita. Int J Mol Sci (2020) 21:7196. doi: 10.3390/ijms21197196

27. Ferreira MS, Kirschner M, Halfmeyer I, Estrada N, Xicoy B, Isfort S, et al. Comparison of Flow-FISH and MM-qPCR Telomere Length Assessment Techniques for the Screening of Telomeropathies. Ann N Y Acad Sci (2020) 1466:93-103. doi: 10.1111 /nyas. 14248

28. Koch A, Voigt S, Kruschinski C, Sanson E, Dückers H, Horn A, et al. Circulating Soluble Urokinase Plasminogen Activator Receptor Is Stably Elevated During the First Week of Treatment in the Intensive Care Unit and Predicts Mortality in Critically Ill Patients. Crit Care (2011) 15:R63. doi: $10.1186 / \mathrm{cc} 10037$

29. Sakaguchi H, Nishio N, Hama A, Kawashima N, Wang X, Narita A, et al. Peripheral Blood Lymphocyte Telomere Length as a Predictor of Response to Immunosuppressive Therapy in Childhood Aplastic Anemia. Haematologica (2014) 99:1312-6. doi: 10.3324/haematol.2013.091165

30. Bai R, Lv Z, Xu D, Cui J. Predictive Biomarkers for Cancer Immunotherapy With Immune Checkpoint Inhibitors. Biomark Res (2020) 8:1-17. doi: 10.1186/s40364-020-00209-0

31. Astuti Y, Wardhana A, Watkins J, Wulaningsih W. Cigarette Smoking and Telomere Length: A Systematic Review of 84 Studies and Meta-Analysis. Environ Res (2017) 158:480-9. doi: 10.1016/j.envres.2017.06.038

32. Woodall MJ, Neumann S, Campbell K, Pattison ST, Young SL. The Effects of Obesity on Anti-Cancer Immunity and Cancer Immunotherapy. Cancers (2020) 12:1230. doi: 10.3390/cancers 12051230

33. Wang Q, Zhan Y, Pedersen NL, Fang F, Hägg S. Telomere Length and AllCause Mortality: A Meta-Analysis. Ageing Res Rev (2018) 48:11-20. doi: 10.1016/j.arr.2018.09.002

34. Weischer M, Nordestgaard BG, Cawthon RM, Freiberg JJ, Tybjærg-Hansen A, Bojesen SE. Short Telomere Length, Cancer Survival, and Cancer Risk in 47102 Individuals. J Natl Cancer Inst (2013) 105:459-68. doi: 10.1093/jnci/dit016

35. Jang RW, Caraiscos VB, Swami N, Banerjee S, Mak E, Kaya E, et al. Simple Prognostic Model for Patients With Advanced Cancer Based on Performance Status. J Oncol Pract (2014) 10:e335-41. doi: 10.1200/JOP.2014.001457

36. Alpert A, Pickman Y, Leipold M, Rosenberg-Hasson Y, Ji X, Gaujoux R, et al. A Clinically Meaningful Metric of Immune Age Derived From HighDimensional Longitudinal Monitoring. Nat Med (2019) 25:487-95. doi: 10.1038/s41591-019-0381-y
37. Jurk D, Wilson C, Passos JF, Oakley F, Correia-Melo C, Greaves L, et al. Chronic Inflammation Induces Telomere Dysfunction and Accelerates Ageing in Mice. Nat Commun (2014) 2:4172. doi: 10.1038/ncomms5172

38. Fyhrquist F, Saijonmaa O, Strandberg T. The Roles of Senescence and Telomere Shortening in Cardiovascular Disease. Nat Rev Cardiol (2013) 10:274-83. doi: 10.1038/nrcardio.2013.30

39. Lian J, Yue Y, Yu W, Zhang Y. Immunosenescence: A Key Player in Cancer Development. J Hematol Oncol (2020) 13:151. doi: 10.1186/s13045-02000986-z

40. Hohensinner PJ, Goronzy JJ, Weyand CM. Telomere Dysfunction, Autoimmunity and Aging. Aging Dis (2011) 2:524-37.

41. Blank CU, Haining WN, Held W, Hogan PG, Kallies A, Lugli E, et al. Defining 'T Cell Exhaustion'. Nat Rev Immunol (2019) 19:665-74. doi: 10.1038/s41577019-0221-9

42. Boeck C, Salinas-Manrique J, Calzia E, Radermacher P, von Arnim CA, Dietrich DE, et al. Targeting the Association Between Telomere Length and Immuno-Cellular Bioenergetics in Female Patients With Major Depressive Disorder. Sci Rep (2018) 8:9419. doi: 10.1038/s41598-018-26867-7

Conflict of Interest: The authors declare that the research was conducted in the absence of any commercial or financial relationships that could be construed as a potential conflict of interest.

Publisher's Note: All claims expressed in this article are solely those of the authors and do not necessarily represent those of their affiliated organizations, or those of the publisher, the editors and the reviewers. Any product that may be evaluated in this article, or claim that may be made by its manufacturer, is not guaranteed or endorsed by the publisher.

Copyright (๑ 2021 Rolles, Gorgulho, Tometten, Roderburg, Vieri, Abels, Vucur, Heymann, Tacke, Brümmendorf, Luedde, Beier and Loosen. This is an openaccess article distributed under the terms of the Creative Commons Attribution License (CC BY). The use, distribution or reproduction in other forums is permitted, provided the original author(s) and the copyright owner(s) are credited and that the original publication in this journal is cited, in accordance with accepted academic practice. No use, distribution or reproduction is permitted which does not comply with these terms. 